\title{
ON THE YOUNG-FENCHEL TRANSFORM FOR CONVEX FUNCTIONS
}

\author{
GERALD BEER
}

(Communicated by R. Daniel Mauldin)

\begin{abstract}
Let $\Gamma(X)$ be the proper lower semicontinuous convex functions on a reflexive Banach space $X$. We exhibit a simple Vietoris-type topology on $\Gamma(X)$, compatible with Mosco convergence of sequences of functions, with respect to which the Young-Fenchel transform (conjugate operator) from $\Gamma(X)$ to $\Gamma\left(X^{*}\right)$ is a homeomorphism. Our entirely geometric proof of the bicontinuity of the transform halves the length of Mosco's proof of sequential bicontinuity, and produces a stronger result for nonseparable spaces.
\end{abstract}

1. Introduction. Let $\Gamma(X)$ denote the proper lower semicontinuous convex functions on a normed linear space $X$. Without question, for reflexive $X$, the fundamental notion of convergence for sequences in $\Gamma(X)$ is Mosco convergence, introduced by U. Mosco in [12].

DEFINITION. Let $X$ be a normed linear space. A sequence of lower semicontinuous proper convex functions $\left\langle f_{n}\right\rangle$ on $X$ is declared Mosco convergent to $f \in \Gamma(X)$ provided at each $x$ in $X$.

(i) there exists a sequence $\left\langle x_{n}\right\rangle$ convergent strongly to $x$ for which $\lim f_{n}\left(x_{n}\right)=$ $f(x)$, and

(ii) whenever $\left\langle x_{n}\right\rangle$ converges weakly to $x$, then $\liminf f_{n}\left(x_{n}\right) \geq f(x)$.

The importance of Mosco convergence in the reflexive setting stems from its stability with respect to duality. With this notion of convergence, the Young-Fenchel transform, i.e., the conjugate operator, is "continuous": if $\left\langle f_{n}\right\rangle$ is Mosco convergent to $f$, then $\left\langle f_{n}^{*}\right\rangle$ is Mosco convergent to $f^{*}([\mathbf{1 3}, \mathbf{7}]$, and in finite dimensions, [16 and 15]).

Mosco convergence of functions is really a special case of a notion of set convergence, identifying elements of $\Gamma(X)$ with their epigraphs, as introduced by Mosco [12, Lemma 1.10]. Specifically, a sequence $\left\langle C_{n}\right\rangle$ of closed convex sets in a reflexive space $X$ is declared Mosco convergent to a closed convex set $C$ provided (i) at each $x$ in $C$ there exists a sequence $\left\langle x_{n}\right\rangle$ convergent strongly to $x$ such that for each $n, x_{n} \in C_{n}$, and (ii) whenever $\langle n(i)\rangle$ is an increasing sequence of positive integers and $x_{n(i)} \in C_{n(i)}$ for each $i$, then the weak convergence of $\left\langle x_{n(i)}\right\rangle$ to $x \in X$ implies $x \in C$. In finite dimensions this reduces to Kuratowski convergence of sets, familiar to any point-set topologist [9]. For further information on Mosco convergence and its applications, one may consult [ 1 or 14$]$.

Received by the editors February 9, 1988.

1980 Mathematics Subject Classification (1985 Revision). Primary 26B25; Secondary 54B20, 46B10.

Key words and phrases. Convex function, conjugate convex function, Young-Fenchel transform, Mosco convergence, Mosco topology, hyperspace. 
In [2], this author introduced a simple geometrically defined topology $\tau_{M}$ on the nonempty closed convex subsets $\mathscr{C}(X)$ of a normed linear space $X$ compatible with Mosco convergence of sequences in $\mathscr{C}(X)$, without reflexivity [2, Theorem 3.1]. (The reader may also consult [1], where a completely different analytical approach to topologizing Mosco convergence of sequences may be found.) When $X$ is reflexive, the topology is Hausdorff and completely regular [2, Theorem 3.4]. To describe this topology, we need some notation. For each $E \subset X$, we introduce these subsets $E^{-}$and $E^{+}$of $\mathscr{C}(X)$

$$
E^{-}=\{C \in \mathscr{C}(X): C \cap E \neq \varnothing\} \quad \text { and } \quad E^{+}=\{C \in \mathscr{C}(X): C \subset E\} .
$$

DEFINITION. Let $\mathscr{C}(X)$ be the nonempty closed convex subsets of a normed linear space $X$. The Mosco topology $\tau_{M}$ on $\mathscr{C}(X)$ is the topology generated by all sets of the form $V^{-}$where $V$ is open in $X$ and $\left(K^{C}\right)^{+}$where $K$ is a weakly compact subset of $X$.

Intuitively, $V^{-}$consists of those convex sets that "hit" $V$, whereas $\left(K^{C}\right)^{+}$consists of those convex that "miss" the weakly compact set $K$. There are several other such "hit-and-miss" topologies in the literature $[\mathbf{2 , ~ 3 , ~ 4 , ~ 8 ] . ~ O f ~ g r e a t e s t ~ i n t e r e s t ~ t o ~}$ topologists is the much stronger finite or Vietoris topology [10], which, in terms of applications, is highly pathological. When $X$ is reflexive, it can be shown that the topology $\tau_{M}$ is first countable if and only if $X$ is separable. But much more comes with separability: $\left\langle\mathscr{C}(X), \tau_{M}\right\rangle$ is a Polish space, i.e., the hyperspace is completely metrizable and separable [2, Theorem 4.3].

Since lower semicontinuous functions have closed epigraphs, we may view $\Gamma(X)$ as a topological subspace of $\left\langle\mathscr{C}(X \times R), \tau_{M}\right\rangle$. As such, a subbase for the Mosco topology on $\Gamma(X)$ consists of all sets of the form $V^{-} \cap \Gamma(X)$ where $V$ is open in $X \times R$ and $\left(K^{C}\right)^{+} \cap \Gamma(X)$ where $K$ is a weakly compact subset of $X \times R$. As a special case of the compatability of Mosco convergence of sequences of convex sets with the topology $\tau_{M}$, Mosco convergence of sequences in $\Gamma(X)$ is compatible with $\tau_{M}$ on $\mathscr{C}(X \times R)$, identifying functions with their epigraphs.

Again suppose that $X$ is reflexive. Since the space $\left\langle\Gamma(X), \tau_{M}\right\rangle$ is first countable if and only if $X$ is separable, Mosco's "continuity" theorem for the Young-Fenchel transform is really only a sequential continuity theorem unless $X$ is separable, for only then do sequences determine the topology. In this note, we show that the transform is actually continuous from $\left\langle\Gamma(X), \tau_{M}\right\rangle$ to $\left\langle\Gamma\left(X^{*}\right), \tau_{M}\right\rangle$. Our proof is entirely geometric, and there is no need to consider limits inferior and limits superior of nets of sets, either explicitly or implicitly, via Lemma 1.10 of [12]. Even more attractive is the shortness of our proof, as compared with Mosco's proof. Finally, we follow Mosco's path to establish a true continuity theorem for the polar operation.

2. Preliminaries and additional notation. In the sequel, $X$ will be a normed linear space with continuous dual $X^{*}$, often reflexive. The origin and closed unit ball of $X\left(\operatorname{resp} . X^{*}\right)$ will be represented by $\theta$ and $B\left(\operatorname{resp} . \theta^{*}\right.$ and $\left.B^{*}\right)$. If $C \in \mathscr{C}(X)$ its polar $C^{\circ}$ is the following subset of $X^{*}$ :

$$
C^{\circ}=\left\{y \in X^{*}: \text { for each } x \in C,\langle y, x\rangle \leq 1\right\} .
$$

We denote the projection map $(x, \alpha) \rightarrow x$ from $X \times R$ to $X$ by $\pi$. 
The epigraph of a convex function $f: X \rightarrow[-\infty, \infty]$ is the following convex subset of $X \times R$ :

$$
\text { epi } f=\{(x, \alpha): x \in X, \alpha \in R \text {, and } \alpha \geq f(x)\} .
$$

Such a set is a closed subset of $X \times R$ if and only if $f$ is lower semicontinuous [5, p. 103]. Dually, the hypograph of $f$ is

$$
\text { hypo } f=\{(x, \alpha): x \in X, \alpha \in R \text {, and } \alpha \leq f(x)\} \text {. }
$$

A convex function $f: X \rightarrow[-\infty, \infty]$ is called proper provided its epigraph is nonempty and contains no vertical lines. As mentioned earlier, $\Gamma(X)$ will denote the proper lower semicontinuous convex functions on $X$. Aff $(X)$ will denote the continuous real affine functions on $X$. If $C \in \mathscr{C}(X)$, then its indicator function $I(\cdot, C)$, defined by

$$
I(x, C)= \begin{cases}0 & \text { if } x \in C, \\ \infty & \text { if } x \notin C\end{cases}
$$

is in $\Gamma(X)$, whereas the support function $s(\cdot, C)$ of $C$, defined by

$$
s(y, C)=\sup \{\langle y, x\rangle: x \in C\} \quad\left(y \in X^{*}\right)
$$

is in $\Gamma\left(X^{*}\right)$. If $f \in \Gamma(X)$ and $\alpha \in R$, we denote its sublevel set at height $\alpha$, i.e., $\{x \in X: f(x) \leq \alpha\}$, by $\operatorname{sub}(f ; \alpha)$.

For each $f \in \Gamma(X)$, we define its conjugate $f^{*}: X^{*} \rightarrow[-\infty, \infty]$ by the formula

$$
f^{*}(y)=\sup \{\langle y, x\rangle-f(x): x \in X\} \text {. }
$$

It is well known that $f^{*} \in \Gamma\left(X^{*}\right)$, and $f^{* *}=f$, provided $X$ is reflexive [6, §14]. We will repeatedly use this fundamental fact: $(y, \alpha) \in$ epi $f^{*}$ if and only if $f$ majorizes the continuous affine functional $x \rightarrow\langle y, x\rangle-\alpha$. The map $f \rightarrow f^{*}$ is called the Young-Fenchel transform.

We will need a slightly different description of the Mosco topology on $\Gamma(X)$, as provided next.

LEMMA 2.1. Let $X$ be a normed linear space. The Mosco topology $\tau_{M}$ on $\Gamma(X)$ is generated by all sets of the form $\Gamma(X) \cap(W \times(-\infty, \alpha))^{-}$where $W$ is open in $X$ and $\Gamma(X) \cap\left(K^{C}\right)^{+}$where $K$ is a weakly compact subset of $X \times R$.

Proof. Suppose $f \in \Gamma(X) \cap V^{-}$where $V$ is open in $X \times R$. Choose $(x, \beta) \in$ hypo $f \cap V$. Since $V$ is open, there exists $\alpha>\beta$ and a neighborhood $W$ of $x$ with $W \times\{\alpha\} \subset V$. Then $f \in(W \times(-\infty, \alpha))^{-} \subset V^{-}$, because epigraphs recede in the upward direction.

LEMMA 2.2. Let $X$ be a normed linear space, and let $f \in \Gamma(X) \cap\left(K^{C}\right)^{+}$, where $K$ is a weakly compact subset of $X \times R$. Then there is a finite subset $\left\{a_{1}, a_{2}, \ldots, a_{n}\right\}$ of $\operatorname{Aff}(X)$ and $\varepsilon>0$ such that for each $i \leq n$,

$$
\inf _{x \in X} f(x)-a_{i}(x)>\varepsilon \quad \text { and } \quad \sup a_{i} \in\left(K^{C}\right)^{+} .
$$

PROOF. Let $k=\left(x_{0}, \alpha_{0}\right)$ be an arbitrary element of $K$. Since $f$ is the supremum of the continuous affine functionals that it majorizes $\left[5\right.$, p. 114], there exists $a_{k} \in$ $\operatorname{Aff}(X)$ with

$$
\inf _{x \in X} f(x)-a_{k}(x)>0 \text { and } a_{k}\left(x_{0}\right)>\alpha_{0} .
$$


The second condition means that $k$ lies in the interior of the hypograph of $a_{k}$, a weakly open subset of $X \times R$ because $a_{k}$ is continuous and affine. By the weak compactness of $K$, there exists $k(1), k(2), \ldots, k(n)$ in $K$ with

$$
K \subset \bigcup_{i=1}^{n} \operatorname{int}\left(\operatorname{hypo} a_{k(i)}\right) \text {. }
$$

Since for each $i \in\{1,2, \ldots, n\}$ we have $\inf _{x \in X} f(x)-a_{k(i)}(x)>0$, there exists $\varepsilon>0$ such that for each $i \in\{1,2, \ldots, n\}$ and each $x \in X, f(x)-a_{k(i)}(x)>\varepsilon$. The desired family of affine functionals is thus $\left\{a_{k(i)}: 1 \leq i \leq n\right\}$.

Lemma 2.2 yields a topological version of the fact that a lower semicontinuous proper convex function is the supremum of the continuous affine functionals that it majorizes (see also $§ 3.5 .2$ of $[1]$ ).

THEOREM 2.3. Let $X$ be a normed linear space, and let $f \in \Gamma(X)$. Let $\Omega$ be the finite subsets of hypo $f^{*}$, ordered by inclusion. For each $F=\left\{\left(y_{1}, \alpha_{1}\right),\left(y_{2}, \alpha_{2}\right), \ldots\right.$, $\left.\left(y_{n}, \alpha_{n}\right)\right\}$ in $\Omega$, define $h_{F} \in \Gamma(X)$ by $h_{F}(x)=\sup _{1 \leq i \leq n}\left\langle y_{i}, x\right\rangle-\alpha_{i}$. Then $F \rightarrow h_{F}$ is an increasing net in $\Gamma(X)$ that is $\tau_{M}$-convergent to $f$.

We also require a general continuity result, which is surely known in some form. The proof is left to the reader.

LEMMA 2.4. Let $X$ be a normed linear space, and let $C_{B}(X, R)$ be the norm continuous real valued functions on $X$ that are bounded on bounded subsets of $X$, equipped with the (locally convex metrizable) topology of uniform convergence on bounded subsets of $X$. Then if $X^{*}$ is equipped with the norm topology, $\varphi: X^{*} \times R \rightarrow$ $C_{B}(X, R)$ defined by $\varphi(y, \alpha)(x)=\langle y, x\rangle-\alpha$ is continuous.

\section{Results.}

THEOREM 3.1. Let $X$ be a reflexive Banach space. Then the Young-Fenchel transform $f \rightarrow f^{*}$ is a homeomorphism of $\left\langle\Gamma(X), \tau_{M}\right\rangle$ onto $\left\langle\Gamma\left(X^{*}\right), \tau_{M}\right\rangle$.

PROOF. Since $f \rightarrow f^{*}$ is an involution, it suffices to show that the transform $f^{*} \rightarrow f$ is continuous. To this end, we show that the inverse image of each subbasic open set in $\left\langle\Gamma(X), \tau_{M}\right\rangle$ is open in $\left\langle\Gamma\left(X^{*}\right), \tau_{M}\right\rangle$.

Suppose $K$ is a weakly compact subset of $X \times R$, and $f \in\left(K^{C}\right)^{+}$, i.e., epi $f \cap K=$ $\varnothing$. By Lemma 2.2 , there exist continuous affine functionals $\left\{a_{1}, a_{2}, \ldots, a_{n}\right\}$ on $X$ and $\varepsilon>0$ such that for each $i \leq n$,

$$
\inf _{x \in X} f(x)-a_{i}(x)>\varepsilon \quad \text { and } \quad \sup a_{i} \in\left(K^{C}\right)^{+} .
$$

For each index $i$, let $y_{i} \in X^{*}$ and $\alpha_{i} \in R$ represent the affine function $a_{i}+\varepsilon$, in that for all $x \in X$,

$$
a_{i}(x)+\varepsilon=\left\langle y_{i}, x\right\rangle-\alpha_{i} .
$$

Since $\pi(K)$ is weakly compact and therefore norm bounded, by Lemma 2.4 , for each index $i$ there exists a norm neighborhood $U_{i}$ of $\left(y_{i}, \alpha_{i}\right)$ such that for all $(y, \delta) \in U_{i}$ for all $x \in \pi(K)$, we have

$$
\left|\langle y, x\rangle-\delta-\left(\left\langle y_{i}, x\right\rangle-\alpha_{i}\right)\right|<\varepsilon
$$

By equation (1), $\left(y_{i}, \alpha_{i}\right) \in$ epi $f^{*}$ for each $i \in\{1,2, \ldots, n\}$, and it follows that $\bigcap_{i=1}^{n} U_{i}^{-}$is a $\tau_{M}$-neighborhood of $f^{*}$ in $\Gamma\left(X^{*}\right)$. We claim that if $h^{*} \in \bigcap_{i=1}^{n} U_{i}^{-}$, 
then $h \in\left(K^{C}\right)^{+}$. To see that epi $h$ does not meet $K$, we show that each point $\left(x_{0}, \alpha_{0}\right)$ of $K$ is not in the epigraph of $h$. By the choice of the affine functionals $\left\{a_{i}: 1 \leq i \leq n\right\}$, there exists an index $i$ such that $\alpha_{0}<a_{i}\left(x_{0}\right)$. Choose $(y, \delta) \in$ epi $h^{*} \cap U_{i}$. This means that $\sup _{x \in X} h(x)-(\langle y, x\rangle-\delta) \geq 0$, and, in particular, $h\left(x_{0}\right) \geq\left\langle y, x_{0}\right\rangle-\delta$. But by the choice of $U_{i}$, we have

$$
\left\langle y, x_{0}\right\rangle-\delta>\left\langle y_{i}, x_{0}\right\rangle-\alpha_{i}-\varepsilon=a_{i}\left(x_{0}\right)>\alpha_{0} .
$$

As a result, $h\left(x_{0}\right)>\alpha_{0}$, so that $\left(x_{0}, \alpha_{0}\right)$ fails to lie in epi $h$. This proves that $h \in\left(K^{C}\right)^{+}$, provided $h^{*} \in \bigcup_{i=1}^{n} U_{i}^{-}$.

To complete the proof, by Lemma 2.1, it suffices to show that whenever $W$ is a norm open subset of $X$, then the inverse image of $(W \times(-\infty, \beta))^{-}$under $f^{*} \rightarrow f$ is $\tau_{M}$-open in $\Gamma\left(X^{*}\right)$. Suppose $f \in(W \times(-\infty, \beta))^{-}$; this means that for some $x_{0} \in W$, we have $f\left(x_{0}\right)<\beta$. Choose $\varepsilon \in(0,1)$ such that $x_{0}+\varepsilon B \subset W$. Also, pick a scalar $\mu$ satisfying

$$
f\left(x_{0}\right)<\mu<\min \left\{\beta, f\left(x_{0}\right)+1\right\} .
$$

Since epi $f=$ epi $f^{* *}$, the choice of $\mu$ ensures

(i) $f^{*}(y)>\left\langle y, x_{0}\right\rangle-\mu$ for each $y \in X^{*}$, and

(ii) there exists $y_{0}$ in $X^{*}$ with $f^{*}\left(y_{0}\right)<\left\langle y_{0}, x_{0}\right\rangle-\mu+1$.

Denote the affine functional $y \rightarrow\left\langle y, x_{0}\right\rangle-\mu$ on $X^{*}$ by $a_{0}$. Also, let $\lambda=$ $\max \left\{\left\|y_{0}\right\|, 4 / \varepsilon\right\}$, and let $K$ be that part of the graph of $a_{0}$ within the vertical cylinder $\left\{(y, \alpha): y \in y_{0}+\lambda B^{*}\right\}$. As $K$ is the intersection of two closed convex sets (the graph and the cylinder), $K$ is a closed convex set, and since $a_{0}$ is Lipschitz, its graph restricted to $y_{0}+\lambda B^{*}$ is a bounded subset of $X^{*} \times R$. Thus, $K$ is a weakly compact subset of the reflexive space $X^{*} \times R$ disjoint from epi $f^{*}$. By (ii), there is an open neighborhood $U$ of $y_{0}$ contained in $y_{0}+B^{*}$ such that for each $y \in U$, we have

$$
f^{*}\left(y_{0}\right)-1<\left\langle y, x_{0}\right\rangle-\mu .
$$

Clearly,

$$
f^{*} \in\left(K^{C}\right)^{+} \cap\left(U \times\left(-\infty, f^{*}\left(y_{0}\right)+1\right)\right)^{-} .
$$

We show that if $h^{*} \in\left(K^{C}\right)^{+} \cap\left(U \times\left(-\infty, f^{*}\left(y_{0}\right)+1\right)\right)^{-}$, then $h \in$ $(W \times(-\infty, \beta))^{-}$. Choose $y_{1} \in U$ with $h^{*}\left(y_{1}\right)<f^{*}\left(y_{0}\right)+1$. Since $K$ is a weakly compact convex set disjoint from the closed convex set epi $h^{*}$, the sets $K$ and epi $h^{*}$ are a positive distance apart, whence $K$ and epi $h^{*}$ can be strongly separated by a closed hyperplane in $X^{*} \times R$. This hyperplane is not vertical, for since $\lambda>4$ and $\left\|y_{0}-y_{1}\right\| \leq 1$, we have $y_{1} \in \pi(K) \cap \pi($ epi $h)$. Thus, the hyperplane is the graph of a continuous affine functional on $X^{*}$, say, $a(y)=\langle y, x\rangle-\alpha$. Since $h^{*}$ majorizes $a,(x, \alpha) \in$ epi $h^{* *}=$ epi $h$.

We claim that $(x, \alpha) \in W \times(-\infty, \beta)$. The idea of the proof is twofold: First, since $\pi(K)$ contains the origin of $X^{*}$, we have $\left(\theta^{*},-\mu\right) \in K$. The point $\left(\theta^{*},-\mu\right)$ must thus lie below $\left(\theta^{*}, a\left(\theta^{*}\right)\right)$, so that $\alpha<\mu$. Second, the gap between epi $h^{*}$ and $K$ at a point near the center of the disc $K$ is narrow relative to the width of $K$, so that the graph of the affine function $a$ must be nearly parallel to the graph of $a_{0}$. Analytically, this means that $\left\|x-x_{0}\right\|$ is small. The details now follow.

Since $\lambda \geq\left\|y_{0}\right\|$, we see that $\theta^{*} \in y_{0}+\lambda B^{*}$, whence $\left(\theta^{*}, a_{0}\left(\theta^{*}\right)\right)=\left(\theta^{*},-\mu\right) \in K$. As a result, $a\left(\theta^{*}\right)=-\alpha$ must exceed $-\mu$, so that $\alpha<\mu<\beta$. We intend to show that $\left\|x-x_{0}\right\| \leq \varepsilon$. Suppose, to the contrary that $\left\|x-x_{0}\right\|>\varepsilon$. By reflexivity, there 
is direction of steepest descent for the functional $x-x_{0}$, i.e., a unit vector $w \in X^{*}$ with $\left\langle w, x-x_{0}\right\rangle=-\left\|x-x_{0}\right\|$. Set $y_{2}=\left(y_{1}+(\lambda / 2) w\right)$. Since $\lambda \geq 4 / \varepsilon>4$, we have

$$
\left\|y_{0}-y_{2}\right\| \leq\left\|y_{0}-y_{1}\right\|+\lambda / 2<1+\lambda / 2<\lambda,
$$

so that $y_{2}$ lies in $\pi(K)$. We show $\left(y_{2}, a\left(y_{2}\right)\right)$ lies below $K$, contradicting the (strong) separation of $K$ from epi $h^{*}$ by the graph of $a$. We have

$$
\begin{aligned}
a\left(y_{2}\right)-a_{0}\left(y_{2}\right) & =\left\langle y_{1}+(\lambda / 2) w, x\right\rangle-\alpha-\left\langle y_{1}+(\lambda / 2) w, x_{0}\right\rangle+\mu \\
& =\left\langle(\lambda / 2) w, x-x_{0}\right\rangle+\left\langle y_{1}, x-x_{0}\right\rangle-\alpha+\mu \\
& =-(\lambda / 2)\left\|x-x_{0}\right\|+a\left(y_{1}\right)-\left\langle y_{1}, x_{0}\right\rangle+\mu .
\end{aligned}
$$

Since $h^{*}$ majorizes $a$, and $y_{1} \in U$ and $h^{*}\left(y_{1}\right) \in\left(-\infty, f^{*}\left(y_{0}\right)+1\right)$, inequality (2) yields

$$
a\left(y_{1}\right) \leq h^{*}\left(y_{1}\right)<f^{*}\left(y_{0}\right)+1<\left\langle y_{1}, x_{0}\right\rangle-\mu+2 .
$$

By assumption, $\left\|x-x_{0}\right\|>\varepsilon$, and since $\lambda>4 / \varepsilon$, formulas (3) and (4) together yield

$$
a\left(y_{2}\right)-a_{0}\left(y_{2}\right)<(-1 / 2)(4 / \varepsilon) \varepsilon+2=0 .
$$

Having obtained the desired contradiction, we conclude that $\left\|x-x_{0}\right\| \leq \varepsilon$, so that

$$
(x, \alpha) \in \operatorname{epi} h \cap\left(\left(x_{0}+\varepsilon B\right) \times(-\infty, \beta)\right) \subset \operatorname{epi} h \cap(W \times(-\infty, \beta)),
$$

completing the proof of the continuity of the Young-Fenchel transform.

Using his sequential continuity theorem, Mosco established the sequential continuity of the polar operation from $\mathscr{C}(X)$ to $\mathscr{C}\left(X^{*}\right)$ by showing that Mosco convergence of a sequence in $\Gamma(X)$ ensures Mosco convergence of sublevel sets above a minimal height. It seems worthwhile to extend his polar result to a legitimate continuity theorem. The geometrical simplicity of the proof is indeed startling.

LEMMA 3.2. Let $X$ be a reflexive Banach space, and suppose $\left\langle f_{\lambda}\right\rangle$ is a net in $\Gamma(X) \tau_{M}$-convergent to $f \in \Gamma(X)$. Then for each $\alpha>\inf f$, we have $\operatorname{sub}(f ; \alpha)=$ $\tau_{M}-\lim \operatorname{sub}\left(f_{\lambda} ; \alpha\right)$.

Proof. Let $\alpha>\inf f$ be fixed. Suppose $W$ is open in $X$ and $\operatorname{sub}(f ; \alpha) \in W^{-}$. Choose $x \in W$ with $f(x) \leq \alpha$. Since $\alpha>\inf f$, there exists $x_{1} \in X$ with $f\left(x_{1}\right)<\alpha$. Since the line segment joining $(x, f(x))$ to $\left(x_{1}, f\left(x_{1}\right)\right)$ lies in epi $f$, it must meet $W \times(-\infty, \alpha)$. As a result, $\left\langle\right.$ epi $\left.f_{\lambda}\right\rangle$ must meet $W \times(-\infty, \alpha)$ eventually, which ensures that $\left\langle\operatorname{sub}\left(f_{\lambda} ; \alpha\right)\right\rangle$ meets $W$ eventually. Suppose now that $\operatorname{sub}(f ; \alpha) \cap K=\varnothing$, where $K$ is a weakly compact subset of $X$. This is equivalent to saying that

$$
\text { epi } f \cap(K \times\{\alpha\})=\varnothing .
$$

But $K \times\{\alpha\}$ is a weakly compact subset of $X \times R$; so, by $\tau_{M}$-convergence of $\left\langle f_{\lambda}\right\rangle$ to $f$, we must have

$$
\text { epi } f_{\lambda} \cap(K \times\{\alpha\})=\varnothing
$$

eventually. Thus, eventually, $\operatorname{sub}\left(f_{\lambda} ; \alpha\right) \cap K=\varnothing$.

THEOREM 3.3. Let $X$ be a reflexive Banach space. Then the polar map $C \rightarrow$ $C^{\circ}$ is a continuous function from $\left\langle\mathscr{C}(X), \tau_{M}\right\rangle$ to $\left\langle\mathscr{C}\left(X^{*}\right), \tau_{M}\right\rangle$.

Proof. Evidently, $C \rightarrow I(\cdot, C)$ is an embedding of $\left\langle\mathscr{C}(X), \tau_{M}\right\rangle$ into $\left\langle\Gamma(X), \tau_{M}\right\rangle$, whence by Theorem $3.1, C \rightarrow I^{*}(\cdot, C)$ is an embedding of $\left\langle\mathscr{C}(X), \tau_{M}\right\rangle$ into 
$\left\langle\Gamma\left(X^{*}\right), \tau_{M}\right\rangle$. But for each $C \in \mathscr{C}(X), I^{*}(\cdot, C)$ is the support functional $s(\cdot, C)$ for $C[6, \S 14]$, and

$$
\inf _{y \in X^{*}} s(y, C) \leq s\left(\theta^{*}, C\right)=0<1 .
$$

Suppose now $C_{1} \in \mathscr{C}(X)$ is fixed, and $\left\langle\mathscr{C}_{\lambda}\right\rangle$ is a net in $\mathscr{C}(X) \tau_{M}$-convergent to $C_{1}$. By Lemma 3.2 and the above remarks, we have

$$
C_{1}^{\circ}=\operatorname{sub}\left(s\left(\cdot, C_{1}\right) ; 1\right)=\tau_{M^{-}} \lim \left[\operatorname{sub}\left(s\left(\cdot, C_{\lambda}\right) ; 1\right)\right]=\tau_{M^{-}} \lim C_{\lambda}^{\circ} .
$$

This establishes $\tau_{M}$-continuity of the polar operation.

All results involving the Young-Fenchel transform untimately rest on the correspondence between the points of the epigraph of $f^{*}$ for a proper lower semicontinuous convex function $f$ and the continuous affine functionals majorized by $f$, given by $(y, \alpha) \rightarrow a(y, \alpha)$, where

$$
a(y, \alpha)(x)=\langle y, x\rangle-\alpha \quad(x \in X) .
$$

Without reflexivity, or even completeness, this is a continuous parametrization. With reflexivity, much more is true.

THEOREM 3.4. Let $X$ be a normed linear space, and let $\psi: X^{*} \times R \rightarrow \operatorname{Aff}(X)$ be defined by $\psi(y, \alpha)=a(y, \alpha)$. Then $\psi$ is continuous, where $X^{*}$ is equipped with the norm topology and $\operatorname{Aff}(X)$ is equipped with the Mosco topology. Moreover, if $X$ is reflexive, then $\psi$ is a homeomorphism.

PROOF. Suppose $a\left(y_{0}, \alpha_{0}\right) \in\left(K^{C}\right)^{+}$where $K$ is a weakly compact subset of $X \times R$. Define $h: \pi(K) \rightarrow R$ by $h(x)=\max \{\beta:(x, \beta) \in K\}$. It is easy to check that $h$ is weakly upper semicontinuous on $K$. Since $a\left(y_{0}, \alpha_{0}\right)(x)>h(x)$ for each $x$ in $K$, and $a\left(y_{0}, \alpha_{0}\right)-h$ is weakly lower semicontinuous, by the weak compactness of $\pi(K), a\left(y_{0}, \alpha_{0}\right)-h$ achieves a minimum positive value on $\pi(K)$. Thus, there exists $\varepsilon>0$ such that for each $x \in \pi(K)$,

$$
a\left(y_{0}, \alpha_{0}\right)(x)>h(x)+\varepsilon \text {. }
$$

Since $\pi(K)$ is weakly compact, it is weakly bounded and is thus norm bounded [5, p. 74]. Applying Lemma 2.4, we see that there exists a norm neighborhood $U$ of $\left(y_{0}, \alpha_{0}\right)$ such that for each $(y, \alpha) \in U$ and each $x \in \pi(K)$, we have $a(y, \alpha)(x)>h(x)$. This means that $a(y, \alpha) \in\left(K^{C}\right)^{+}$.

Next suppose $a\left(y_{0}, \alpha_{0}\right) \in(W \times(-\infty, \beta))^{-}$for some open subset $W$ of $X$. There exists $x_{0} \in W$ with $a\left(y_{0}, \alpha_{0}\right)\left(x_{0}\right)<\beta$. Pick $\varepsilon>0$ such that

$$
a\left(y_{0}, \alpha_{0}\right)\left(x_{0}\right)+\varepsilon<\beta .
$$

If $x_{0}=\theta$, then $a\left(y_{0}, \alpha_{0}\right)\left(x_{0}\right)=-\alpha_{0}$. As a result, if $\left|\alpha-\alpha_{0}\right|<\varepsilon$ and $y$ is arbitrary, then by (5),

$$
a(y, \alpha)\left(x_{0}\right)=a(y, \alpha)(\theta)=-\alpha<\beta
$$

and we have $a(y, \alpha) \in(W \times(-\infty, \beta))^{-}$. If $x_{0} \neq \theta$, we claim that

$$
V \equiv\left(y_{0}+\left(\varepsilon / 2\left\|x_{0}\right\|\right) B^{*}\right) \times\left(\alpha_{0}-\varepsilon / 2, \alpha_{0}+\varepsilon / 2\right)
$$

is mapped by $\psi$ into $(W \times(-\infty, \beta))^{-}$. Suppose $(y, \alpha) \in V$. We have

$$
\left|a(y, \alpha)\left(x_{0}\right)-a\left(y_{0}, \alpha_{0}\right)\left(x_{0}\right)\right| \leq\left\|y-y_{0}\right\| \cdot\left\|x_{0}\right\|+\left|\alpha-\alpha_{0}\right|<\varepsilon / 2+\varepsilon / 2=\varepsilon .
$$


As a consequence of $(5), a(y, \alpha)\left(x_{0}\right)<\beta$, and $a(y, \alpha) \in(W \times(-\infty, \beta))^{-}$, because $\left(x_{0}, a(y, \alpha)\left(x_{0}\right)\right) \in W \times(-\infty, \beta)$. Continuity of $\psi$ is now established, without reflexivity.

We now show that $\psi^{-1}: \operatorname{Aff}(X) \rightarrow X^{*} \times R$ is continuous, provided $X$ is reflexive. This is almost immediate from Theorem 3.1. Fix $\left(y_{0}, \alpha_{0}\right)$ in $X^{*} \times R$, and suppose $\left\langle\left(y_{\lambda}, \alpha_{\lambda}\right)\right\rangle$ is a net in $X^{*} \times R$ such that

$$
a\left(y_{0}, \alpha_{0}\right)=\tau_{M}-\lim a\left(y_{\lambda}, \alpha_{\lambda}\right) .
$$

By Theorem 3.1, $a^{*}\left(y_{0}, \alpha_{0}\right)=\tau_{M}-\lim a^{*}\left(y_{\lambda}, \alpha_{\lambda}\right)$. In terms of epigraphs, this says that $\left\{\left(y_{0}, \beta\right): \beta \geq \alpha_{0}\right\}=\tau_{M}-\lim \left\{\left(y_{\lambda}, \beta\right): \beta \geq \alpha_{\lambda}\right\}$. It is now obvious that $\lim \left\|y_{\lambda}-y_{0}\right\|=0$ and $\lim \alpha_{\lambda}=\alpha_{0}$.

COROLlaRY 3.5. Let $X$ be a reflexive Banach space, and let $f$ be a proper lower semicontinuous convex function on $X$. Then epi $f^{*}$ as a subspace of $X^{*} \times R$ with the norm topology, is homeomorphic to the continuous affine functions majorized by $f$, equipped with the Mosco topology.

COROLlaRY 3.6. Let $X$ be a reflexive Banach space. Then $\left\langle\operatorname{Aff}(X), \tau_{M}\right\rangle$ is completely metrizable.

To conclude, we show that when $X$ is separable and reflexive, we can select for each $f \in \Gamma(X)$ a continuous affine function $a_{f}$ majorized by $f$ in such a manner that $f \rightarrow a_{f}$ is a continuous function on $\left\langle\Gamma(X), \tau_{M}\right\rangle$.

THEOREM 3.7. Let $X$ be a separable reflexive Banach space. Let $f_{1} \in \Gamma(X)$ and let $a_{1}$ be a fixed continuous affine function on $X$ majorized by $f_{1}$. Then there exists $\sigma:\left\langle\Gamma(X), \tau_{M}\right\rangle \rightarrow\left\langle\operatorname{Aff}(X), \tau_{M}\right\rangle$ such that $\sigma$ is continuous, $\sigma\left(f_{1}\right)=a_{1}$, and for each $f \in \Gamma(X)$ and $x \in X$, we have $\sigma(f)(x) \leq f(x)$.

Proof. Suppose $f \in \Gamma(X)$ is arbitrary. By Theorem 3.1, whenever $V$ is an open subset of $X^{*} \times R$ that meets the epigraph of $f^{*}$, there is a $\tau_{M}$-neighborhood of $f$ such that epi $h \cap V \neq \varnothing$ for each $h$ in the neighborhood. Thus, if we view $f \rightarrow$ epi $f^{*}$ as a set valued function from $\Gamma(X)$ to $\mathscr{C}\left(X^{*} \times R\right)$, then this correspondence is lower semicontinuous in the sense of Kuratowski ([8, p. 73; 9, p. 173 and 11]). Clearly, the correspondence has closed convex values. If $a_{1}(x)=\left\langle y_{1}, x\right\rangle-\alpha_{1}$, and we assign $\left\{\left(y_{1}, \alpha_{1}\right)\right\}$ (rather than epi $\left.f_{1}^{*}\right)$ to $f_{1}$, the correspondence remains lower semicontinuous and convex valued. By Theorem 4.3 of $[2],\left\langle\Gamma(X), \tau_{M}\right\rangle$ is metrizable and is thus paracompact. Applying Michael's selection theorem [11], there exists a continuous function $\rho:\left\langle\Gamma(X), \tau_{M}\right\rangle \rightarrow X^{*} \times R$ such that for each $f \in \Gamma(X)$, we have both $\rho(f) \in$ epi $f^{*}$ and $\rho\left(f_{1}\right)=\left(y_{1}, \alpha_{1}\right)$. With $\psi: X^{*} \times R \rightarrow \operatorname{Aff}(X)$ as in the statement of Theorem 3.4, $\sigma=\psi \circ \rho$ is the desired function.

Separability of $X$ is only used to guarantee paracompactness of the function space $\left\langle\Gamma(X), \tau_{M}\right\rangle$. We have no idea whether or not $\left\langle\Gamma(X), \tau_{M}\right\rangle$ is paracompact for a nonseparable reflexive space $X$.

\section{REFERENCES}

1. H. Attouch, Variational convergence for functions and operators, Pitman Publishers, Boston, 1984.

2. G. Beer, On Mosco convergence of convex sets, Bull. Australian Math. Soc. 38 (1988), 239-253. 
3. G. Beer, C. Himmelberg, K. Prikry and F. Van Vleck, The locally finite topology on $2^{X}$, Proc. Amer. Math. Soc. 101 (1987), 168-172.

4. S. Francaviglia, A. Lechicki and S. Levi, Quasi-uniformization of hyperspaces and convergence of nets of semicontinuous multifunctions, J. Math. Anal. Appl. 112 (1985), 347-370.

5. J. Giles, Convex analysis with application in differentation of convex functions, Research Notes in Math., vol. 58, Pitman, London, 1982.

6. R. Holmes, A course in optimization and best approximation, Lecture Notes in Math., vol. 257, Springer-Verlag, New York, 1972.

7. J. Joly, Une famille de topologies sur l'ensemble des fonctions convexes pour lesquelles la polarité est bicontinue, J. Math. Pures Appl. 52 (1973), 421-441.

8. E. Klein and A: Thompson, Theory of correspondences, Wiley, Toronto, 1984.

9. K. Kuratowski, Topology, vol. 1, Academic Press, New York, 1966.

10. E. Michael, Topologies on spaces of subsets, Trans. Amer. Math. Soc. 71 (1951), 152-182.

11. $\ldots$ Selected selection theorems, Amer. Math. Monthly 63 (1956), 233-238.

12. U. Mosco, Convergence of convex sets and of solutions of variational inequalities, Adv. in Math. 3 (1969), 510-585.

13. _ 518-535.

14. Y. Sonntag, Convergence au sens de Mosco; théorie et applications à l'approximation des solutions d'inéquations, Thèse d'Etat, Université de Provence, Marseille, 1982.

15. D. Walkup and R. Wets, Continuity of some convex-cone valued mappings, Proc. Amer. Math. Soc. 18 (1967), 229-235.

16. R. Wijsman, Convergence of sequences of convex sets, cones, and functions. II, Trans. Amer. Math. Soc. 123 (1966), 32-45.

Department of Mathematics, California State University, Los Angeles,
CALifornia 90032 